\title{
Experience and outcome of 3 years of a European EQA scheme for genetic testing of the spinocerebellar ataxias
}

\author{
Sara Seneca ${ }^{1}$, Michael A Morris ${ }^{2}$, Simon Patton ${ }^{3}$, Rob Elles ${ }^{3}$ and Jorge Sequeiros ${ }^{*}, 4$ \\ ${ }^{1}$ Center of Medical Genetics, University Hospital Brussel (UZ Brussel), Vrije Universiteit Brussel (VUB), Brussels, \\ Belgium; ${ }^{2}$ Laboratoire de Diagnostic Moléculaire, Service de Médecine Génétique, University Hospitals, Geneva, \\ Switzerland; ${ }^{3}$ European Molecular Genetics Quality Network (EMQN), National Genetics Reference Laboratory, \\ St Mary's Hospital, Manchester, UK; ${ }^{4}$ UnIGENe and CGPP, IBMC - Instituto de Biologia Molecular e Celular and \\ ICBAS, Universidade do Porto, Porto, Portugal
}

The European Molecular Genetics Quality Network (EMQN) has been organizing an external quality assessment (EQA) scheme for molecular genetic testing of trinucleotide repeat mutations in the spinocerebellar ataxias (SCAs) since 2004. DNA samples were validated by at least two independent labs and two different methods. Together with mock clinical case descriptions and requests for specific SCA gene analyses, these were sent to registered participants each year. Laboratories were asked to use their routine procedures and protocols. A panel of assessors reviewed the final returns, including genotype results and reports, to assess the quality of (1) genotyping and (2) interpretation and reporting. A description of methods and raw data were also requested and were very useful for the final analysis. Altogether, during 3 years, 239 reports were received from the laboratories. Overall genotype error rate ranged $1.1-5.2 \%$, a significant cause of concern. Scores for interpretation and reporting also showed that there is still much room for progress, although performance has improved over this period of assessment. The consequences of suboptimal laboratory practices, genotyping errors and misdiagnosis and of incorrect or incomplete interpretation and reporting have wide implications for patient lives, as well as for health management and counselling of relatives. EQA schemes are an important part of quality assurance in molecular genetic laboratories, and their use should become a routine part of laboratory diagnostic practice. Current evidence shows also that it is important that laboratories participate on a yearly basis and that this becomes mandatory for reference laboratories.

European Journal of Human Genetics (2008) 16, 913-920; doi:10.1038/ejhg.2008.29; published online 27 February 2008

Keywords: EMQN; proficiency testing; quality assurance; SCA; ataxia

\section{Introduction}

The spinocerebellar ataxias (SCAs) are a clinically and genetically heterogeneous group of neurodegenerative disorders. At least 30 loci for autosomal-dominant SCA

*Correspondence: Dr J Sequeiros, Universidade do Porto, UNIGENE IBMC, R Campo Alegre 823, Porto 4150-180, Portugal.

Tel: + 35122607 4942; Fax: + 35122600 2923;

E-mail: jsequeir@ibmc.up.pt

Received 12 November 2007; revised 20 December 2007; accepted 17 January 2008; published online 27 February 2008 have been mapped, over the past 15 years, and the respective genes identified for about half of these. ${ }^{1}$

SCA affects mainly the central nervous system; patients exhibit a cerebellar syndrome, including gait and limb ataxia, dysarthria and impaired eye movements, often associated with other variable signs and symptoms (spasticity, dystonia, tremor, peripheral amyotrophies, contraction fasciculations, eye lid reaction, chorea, epilepsy, hemiplegic migraine, retinal degeneration, mental retardation, dementia). These may sometimes help in their 
differential clinical diagnosis, although this is not always straightforward: firstly, there is a striking phenotypic overlap among the SCAs; and secondly, each SCA may exhibit considerable clinical variation, including age-atonset, even within a single family.

The worldwide prevalence of the different dominant ataxias is variable and good estimates for specific countries or regions are rarely available. Nevertheless, most epidemiologic studies have shown a prevalence rate between 1.6 and 5.5 in 100000 (in Cantabria, northern Spain and Portugal, respectively). Their relative frequency within a population is usually better known, and can be an important tool for diagnostic laboratories. Worldwide, SCA1, SCA2, Machado-Joseph disease (MJD/SCA3), SCA6 and SCA7 are the most common forms, typically representing $60-70 \%$ of families; depending on the population, one or another of these may be the most frequent. ${ }^{1}$

These five SCAs, plus SCA17 and dentatorubral-pallidoluysian atrophy, are all polyglutamine diseases, caused by the expansion of translated $(\mathrm{CAG})_{n}$ in the coding region of the genes involved. CAG repeats are polymorphic in length in the normal population and become expanded in affected individuals. The expansion size varies among patients, but, most importantly, normal and pathological ranges differ significantly among SCA types. In some, intermediate repeats, interrupted repeats and meiotic instability of pathogenic alleles provide further challenges for diagnostic testing and genetic counselling.

Reliable molecular genetic testing has important implications for patient diagnosis, presymptomatic testing and counselling, as well as for a better scientific understanding and knowledge about disease frequency. Reports from other external quality assessment (EQA) schemes have indicated that genotyping errors or erroneous interpretation of results, leading to misdiagnosis, occur frequently and that current quality standards are in need of significant improvement. $^{2-8}$ EQA objectively assesses laboratory performance, by an independent body, and is widely recognized as a key tool for improving their quality standards. ${ }^{2}$

In 2004, the European Molecular Genetics Quality Network (EMQN) initiated a novel EQA scheme for the SCAs. This aimed at (1) measuring and documenting standards, practices and methodologies used, (2) improving laboratories' performance, and (3) evaluating progress in the quality of molecular genetic testing of the most common SCAs, throughout Europe (and beyond).

We now present the results of this new EQA scheme, for the period 2004-2006.

\section{Materials and methods \\ European Molecular Genetics Quality Network}

EMQN is a not-for-profit organization, promoting quality in molecular genetic testing in Europe and beyond, through the provision of EQA (proficiency testing) schemes, the organization of best practice meetings and the publication of guidelines. It started as an EC 4th Framework Programme project, and is now sustained mainly by the subscription of its members and a small fee for the participation in each scheme. It is also closely linked to the 6th FP NoE EuroGentest.

\section{General procedure}

EQA materials (DNA samples matched to a detailed description of mock clinical situations) were selected by the scheme organizer (JS) and assessors (SS and MAM), to simulate commonly encountered requests for testing, and chosen to be representative in terms of technical requirements and interpretative complexity. Offering similar diagnostic situations in consecutive years was avoided. Marking criteria were discussed and defined by the scheme organizer and assessors, and harmonized, as much as possible, with other EMQN schemes and, in particular, those for other trinucleotide repeat disorders (Huntington disease, Fragile $\mathrm{X}$ syndrome and Friedreich ataxia).

The EQA scheme was advertised every year to the clinical molecular genetics community, through EMQN mailings and its website. The identity of the participating laboratories was blinded to the scheme organizer and assessors; laboratories were assigned code numbers, known only to the EMQN administrator (SP). Laboratories were asked to remove from their reports all information that might identify them and to use only their code number. EQA materials were aliquoted and distributed by EMQN to all participants, by courier, at environment temperature, together with the scheme instructions. Participants were asked to treat cases and samples according to their routine. Individual reports for each case were returned to EMQN before 12 weeks after the distribution of the samples. Reports were accepted in English, French, Spanish or Italian. Participants were also encouraged to provide separate detailed information about methods and primers used, as well as their raw data.

All reports were evaluated and marked by the same assessors, for accuracy of genotyping, as well as for appropriateness of interpretation and reporting of results. An individual report was sent to each participating laboratory, with their final genotyping and reporting scores, as well as the average of all the laboratories enrolled in the scheme; personalized comments and suggestions were added, whenever appropriate. In addition, a final report was sent, showing all the individual scores (anonymous) and including comments on the overall achievements and conclusions from the scheme. Laboratories had the right to appeal for a given period of time, through the EMQN administration.

\section{DNA samples}

Anonymized genomic DNAs, from affected or non-affected individuals, were selected by the scheme organizer for 
seven cases. DNA extracted from cell lines (Coriell, New Jersey, USA) was used for the other two cases. For each EQA sample, aliquots of $5 \mu \mathrm{g}$ of DNA were shipped to each laboratory, in TE solution, at a final concentration of $100 \mu \mathrm{g} / \mathrm{ml}$ (in principle, sufficient to repeat assays two or three times), in labelled $0.5 \mathrm{ml}$ tubes. Due to a problem in sample distribution for case 3, this was excluded from marking in 2005.

Prior to aliquoting, all DNA samples were validated by at least two assessors, using standard procedures for testing of SCA1, SCA2, SCA3/MJD, SCA6 or SCA7 (genes ATXN1, $A T X N 2, A T X N 3, C A C N A 1 A$ or $A T X N 7$, respectively), and by direct sequencing of PCR products. Although this is technically more difficult for expansions, sequencing can allow precise determination of the number of trinucleotides. After capillary electrophoresis or polyacrylamide gel electrophoresis (PAGE), the number of repeat units was estimated by sizing of the PCR fragment, using size standards. Blank (no DNA) and control samples (known repeat sizes) were incorporated in each analysis, to validate the test results. Control samples were chosen appropriately to evaluate repeat tract length across the polymorphic and pathological ranges, as normally encountered in diagnostic routine. For each SCA locus, the normal and pathologic ranges were determined with reference to the most recent literature (Table 1).

\section{Clinical cases}

Every year's scheme consisted of three mock clinical cases, each requesting a diagnostic, presymptomatic or prenatal test. These were intended to be representative of some of the most commonly encountered requests for SCA testing (Table 2).

\section{Report evaluation and marking}

Prior to each year's scheme, the assessors defined marking criteria for genotyping and interpretation/reporting, sent to the laboratories with the cases and samples. Every report was evaluated independently by three assessors. Separate marks (maximum score of 2.00) were given, for accuracy of the genotype and for accuracy and completeness of the report and interpretation. Details for marking and evaluation are outlined in Table 3.

\section{Results}

\section{Participation and returns}

Fifty-two participants, from 22 countries, have enrolled in this programme, during its 3 years. These have included university, hospital, private and research laboratories from Belgium, Canada, Croatia, Cyprus, Czech Republic, Denmark, Estonia, Finland, France, Germany, Hong Kong, Israel, Italy, Norway, Portugal, Singapore, Spain, Sweden, Switzerland, The Netherlands, Turkey and the United Kingdom. Participation, however, was not consistent: only
28.8\% (15/52) laboratories participated for 3 years; $34.6 \%$ (18/52) participated for 2 years, while $36.5 \%(19 / 52)$ participated only once.

Registration and returned results are indicated in Table 4. Unexpected delays and technical difficulties were the main reasons reported for dropout. In addition (in 2006), one lab reported only genotypes, but did not return individual reports; 10 laboratories returned reports for only some of the cases, justifying it with sample reception problems $(1 \times)$, technical (PCR amplification) problems $(7 \times)$, or not offering testing for a particular locus $(1 \times)$; in 2004, one lab sent in the same report twice, thus failing to report on one case. In a few cases, laboratories did not anonymize their reports or did not include their unique EMQN code number on the reports.

\section{Methodology used}

Although a separate detailed description of methodologies was requested, only a few laboratories provided it; however, a vast majority (2004: 84.3\%; 2005: 100\%; 2006: $92.3 \%$ ) described their methods in the reports, as recommended by both reporting guidelines and international accreditation standards (SSMG (Swiss Society of Medical Genetics), 2003; ISO 15189, 2007). No changes in technologies were seen, over the 3 years. The main method was fragment sizing of fluorescently labelled PCR products, using capillary or PAGE. One lab used silver staining and one lab used only agarose gel electrophoresis. Two participants always systematically sequenced the repeats on all EQA samples.

\section{Genotyping performance}

The total number of reports assessed was 275. The overall genotyping standard was good, with $97.5 \%$ of the reports correctly identifying the genotypes as 'normal' or 'pathogenic' (Table 4); however, of the 14 laboratories that submitted all case reports for every year's scheme, only 2 (14.2\%) achieved maximum genotype scores for all cases, due to difficulties felt by others with repeat counting.

Not all laboratories included repeat sizes in their reports: three did not report size of normal alleles, one did not report size of expanded alleles, and seven did not report repeat size at all. Despite the correct assignment of most alleles to the normal or pathogenic range, considerable interlaboratory variation was seen in the reported repeat size, both for normal and expanded alleles, throughout the 3 years. In the 2004 pilot, the majority of participants consistently sized alleles outside the margin of error set, but without this resulting in a misdiagnosis. In addition, three laboratories were unable to differentiate between samples that were homoallelic (homozygous for normal alleles of the same size) and those having two normal alleles separated by a single triplet repeat. Sizing within the limits set improved considerably in the second and third year, except for the SCA7 test, introduced in 2006. In 2004 
Table 1 Normal, pathogenic and uncertainty ranges for SCA loci repeats

\begin{tabular}{|c|c|c|c|c|}
\hline Locus & Normal & Uncertain & Pathogenic & References \\
\hline SCA1 & $6-38$ & $39-42$ & $39-82$ & Orr et $a l^{\rho}$ and Zühlke et $a l^{10}$ \\
\hline DRPLA & $6-34$ & - & $49-88$ & Koide et $a l^{11}$ and Nagafuchi et $a l^{12}$ \\
\hline SCA2 & $15-24$ & $32-34$ & $35-200$ & Imbert et $a l_{1}^{13}$ Pulst et $a l_{,}^{14}$ Sanpei et $a l^{15}$ and Silveira et $a l^{16}$ \\
\hline MJD/SCA3 & $12-41$ & $45-56$ & $61-84$ & Maciel et $a l_{1}^{17}$ Maciel et $a l_{1}^{18}$ Takiyama et $a l^{19}$ and Padiath et $a 2^{20}$ \\
\hline SCA6 & $4-18$ & - & $19-33$ & Zhuchenko et $a^{21}$ \\
\hline SCA7 & $4-27$ & $28-35$ & $37-306$ & David et $a l^{22}$ and Stevanin et $a l^{23}$ \\
\hline SCA8 & $16-34$ & - & $>74$ & Koob et $a 1^{24}$ \\
\hline SCA10 & $10-29$ & $280-370$ & $400-4500$ & Matsuura et $a l^{25}$ and Alonso et $a l^{26}$ \\
\hline SCA12 & $7-45$ & - & $55-78$ & Holmes et $a 1^{27}$ \\
\hline SCA17 & $25-42$ & $43-48$ & $45-63$ & Zühlke et $a 1^{28}$ and Oda et $a l^{29}$ \\
\hline
\end{tabular}

Table 2 DNA samples and three mock clinical cases

\begin{tabular}{|c|c|c|c|c|}
\hline Clinical request & SCA1 & SCA2 & $M J D / S C A 3$ & SCA7 \\
\hline Diagnostic & & Case 2-2006 & $\begin{array}{l}\text { Case } 1-2004 \\
\text { Case } 3-2004 \\
\text { Case } 1-2005\end{array}$ & \\
\hline Presymptomatic & Case $3-2005^{a}$ & $\begin{array}{l}\text { Case } 2-2004 \\
\text { Case } 2-2005\end{array}$ & Case 1-2006 & \\
\hline Prenatal & & & & Case \\
\hline
\end{tabular}

${ }^{\mathrm{a}}$ Case 3-2005 was withdrawn from marking.

Table 3 Marking criteria genotyping and interpretation/reporting

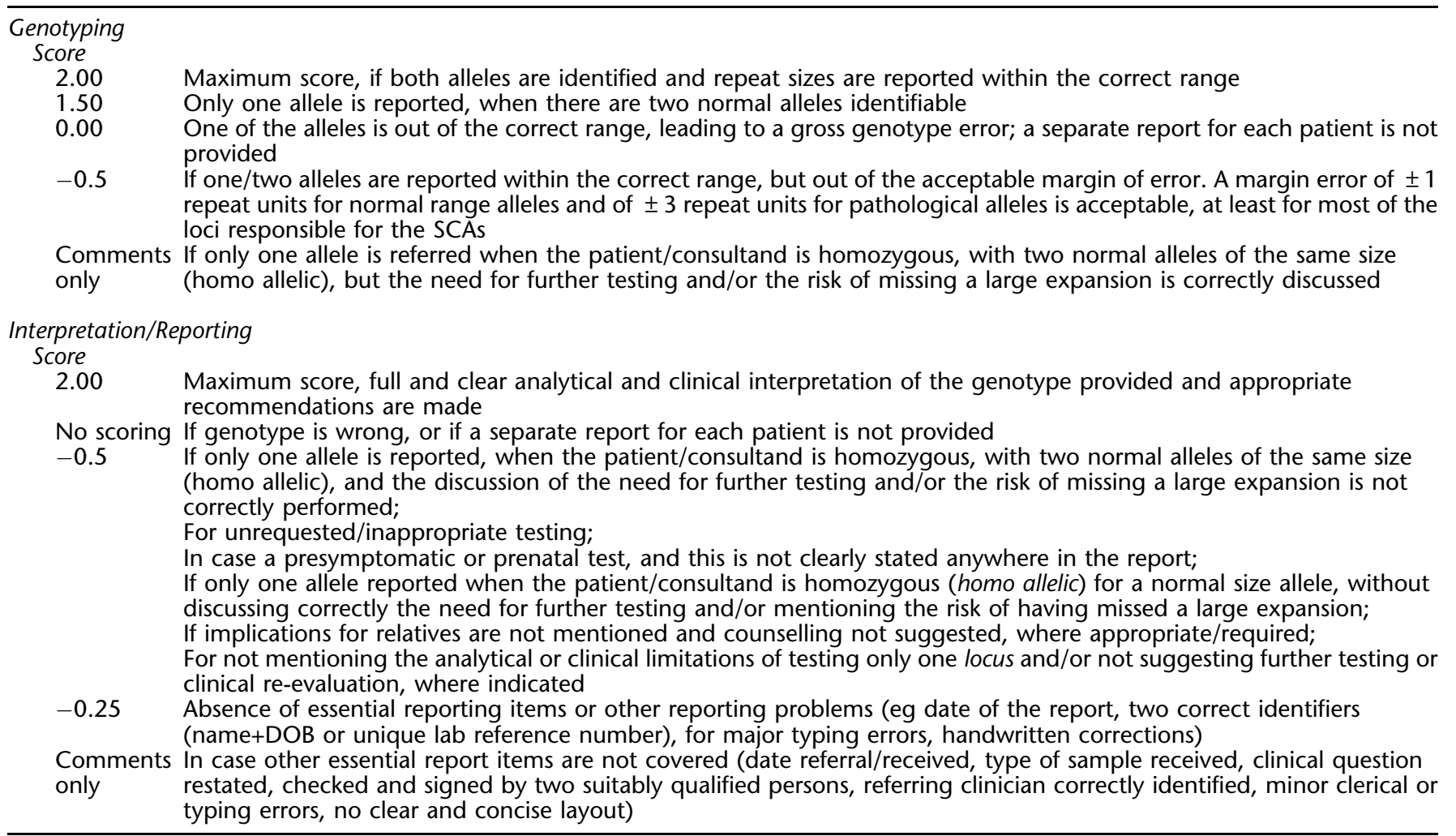

and 2005, genotyping for MJD/SCA3 revealed two distinct approaches: most laboratories included the first six triplets (the unstable region of the ATXN3 gene has the sequence
$(\mathrm{CAG})_{2}$ CAAAAG CAGCAA $\left.(\mathrm{CAG})_{n}\right)$, but others counted only the 'pure' (CAG) $)_{n}$ repeat. In the 2004 and 2005 final scheme reports, we recommended that the count be made 
Table 4 Global results of the SCA scheme for the years 2004-2006

\begin{tabular}{|c|c|c|c|c|}
\hline Participation & 2004 & 2005 & 2006 & Total \\
\hline $\begin{array}{l}\text { Registered labs } \\
\text { Returns (labs) } \\
\text { Returns (genotypes reported) }\end{array}$ & $\begin{array}{l}33 \\
32 \\
94\end{array}$ & $\begin{array}{c}37 \\
37 \\
105(68)^{\mathrm{a}}\end{array}$ & $\begin{array}{l}28 \\
26 \\
76\end{array}$ & $\begin{array}{c}98 \\
95 \\
275\left(238^{a}\right)\end{array}$ \\
\hline $\begin{array}{l}\text { Genotyping scores } \\
\text { Mean score } \\
2.0 / 2.0 \\
0.0 / 2.0\end{array}$ & $\begin{array}{ll} & 1.91 \\
88 & (93.6 \%) \\
1 & (1.1 \%)\end{array}$ & $\begin{array}{c}1.78 \\
35(50.7 \%) \\
2(2.9 \%)\end{array}$ & $\begin{array}{c}1.79 \\
46(59.7 \%) \\
4(5.2 \%)\end{array}$ & $\begin{array}{c}1.83 \\
169(70.4 \%) \\
7(2.9 \%)\end{array}$ \\
\hline $\begin{array}{l}\text { Interpretation scores } \\
\text { Mean score } \\
2.0 / 2.0\end{array}$ & & $\begin{array}{c}1.53 \\
29 / 66(43.9 \%)\end{array}$ & $\begin{array}{c}1.76 \\
31 / 70(44.3 \%)\end{array}$ & $136(44.1 \%)$ \\
\hline
\end{tabular}

according to the scientific literature and that the first six triplets be included. ${ }^{30,17}$ This had the effect of standardizing this practice in the 2006 EQA.

Gross diagnostic errors occurred every year (Table 4). In 2004 and 2005, two participants reported a non-existent expanded allele, in a presymptomatic test for SCA2; in 2005 and 2006, three laboratories failed to detect the pathogenic allele in the diagnostic test for MJD/SCA3 or SCA7; and finally, in 2006, one other laboratory reported a false-negative MJD/SCA3 genotype and a false-positive SCA2 genotype, presumably because of sample misidentification. However, except for this case, no laboratory made more than one error.

Although practice and legislation varies from country to country, testing for SCA loci other than that requested is considered inappropriate, and particularly more so in presymptomatic or prenatal testing. This was a problem identified in the reports from a number of laboratories in the pilot year, a practice that apparently ended, after comments in the 2004 final report.

\section{Interpretation and reporting}

The complexity of genetic testing results and the potential implications for patients and their family members of getting these wrong means that the report of the test results must include an accurate interpretation of the genotype and the adequate recommendations, in the context of the patient and family history and the referral question.

Only a few errors of interpretation, serious enough to be potentially prejudicial to patient care, were observed. One laboratory (in 2006) misinterpreted a correctly genotyped full mutation as an intermediate allele. In 2005, labs did not correctly address the issue of homoallelism: $62 \%$ $(21 / 28)$ of laboratories did not discuss limitations of the methods used, including the possibility of having missed a very large expansion. Another serious problem was the reporting by a few labs (13 over 3-year period) of presymptomatic or prenatal tests as if they were diagnostic, thus providing inadequate interpretation and conclusions.

Clerical errors and mistakes in transcription of the results may have major implications for a patient report. Common errors of this type included using incorrect or incomplete patient identifiers, very often as a direct result of typographic errors in names and dates of birth. Some standard practices, as ticking off a box in a multiple choice setting ('positive/negative'), should be avoided too, as this is not only inadequate but also rather prone to errors. Also frequent (11 over 3 years) were cut-and-paste errors, leading to gross mistakes, such as the wrong disease, locus tested or patient name.

Some less critical but still important errors were often seen in reports. We assessed the presence or absence of a number of key elements of reporting, including essential items such as locus and disorder studied patient identifiers, unique lab number and report date, and other 'best practice' items (Table 5). These are not specific to SCA, but generally applicable to all genetic tests. All elements are present in the reports from the majority of laboratories, and the assessors became aware of a general improvement in reporting practice since the first year of the scheme, thus supporting the educative value of EQA participation.

\section{Discussion}

This is the first report of an EQA scheme for molecular genetic testing of autosomal-dominant SCA. Proficiency testing was performed for SCA1, SCA2, MJD/SCA3, SCA6 and SCA7, the most frequent worldwide. It assessed genotyping accuracy, as well as interpretation and reporting. Participants were provided with purified DNA, and questions included confirmation or exclusion of a clinical diagnosis, presymptomatic and prenatal testing.

In general, its results were encouraging, although gross genotyping errors were detected every year. Sample misidentification, technical failures, misinterpretation of 
Table 5 Reporting items used by the participating laboratories

\begin{tabular}{|c|c|c|c|}
\hline & 2004 & 2005 & 2006 \\
\hline \multicolumn{4}{|l|}{ Identifiers } \\
\hline Full (correct) name & $29 / 32$ & $30 / 37$ & $23 / 26$ \\
\hline $\mathrm{DOB}$ & $27 / 32$ & $36 / 37$ & $25 / 26$ \\
\hline Unique sample number & $25 / 32$ & $33 / 37$ & $20 / 26$ \\
\hline Gender & $13 / 32$ & $21 / 37$ & $17 / 26$ \\
\hline \multicolumn{4}{|c|}{ Reports general information and appearance } \\
\hline Layout clear and concise & $26 / 32$ & $34 / 37$ & $16 / 26$ \\
\hline $\begin{array}{l}\text { No content and cut and paste } \\
\text { errors }\end{array}$ & $26 / 32$ & $35 / 37$ & $23 / 26$ \\
\hline Date of report & $30 / 32$ & $31 / 37$ & $23 / 26$ \\
\hline Two signatures $^{\mathrm{a}}$ & $24 / 32$ & $34 / 37$ & $24 / 26$ \\
\hline Only one page report ${ }^{a}$ & $28 / 32$ & $35 / 37$ & $24 / 26$ \\
\hline No handwritten corrections & $28 / 32$ & $36 / 37$ & $25 / 26$ \\
\hline $\begin{array}{l}\text { No distinction between } \\
\text { presymptomatic, diagnostic or }\end{array}$ & $28 / 32$ & $33 / 37$ & $21 / 26$ \\
\hline prenatal testing & $29 / 32$ & $34 / 37$ & $23 / 26$ \\
\hline $\begin{array}{l}\text { counselling } \\
\text { Implications for relatives }\end{array}$ & $25 / 32$ & $30 / 37$ & $20 / 26$ \\
\hline \multicolumn{4}{|l|}{ Referral information } \\
\hline Locus disease to be tested & $30 / 32$ & $37 / 37$ & $25 / 26$ \\
\hline Type of request & $27 / 32$ & $33 / 37$ & $21 / 26$ \\
\hline Type of sample & $20 / 32$ & $27 / 37$ & $19 / 26$ \\
\hline Date received & $26 / 32$ & $30 / 37$ & $20 / 26$ \\
\hline Referring physician & $27 / 32$ & $33 / 37$ & $21 / 26$ \\
\hline Referral reasons & $25 / 32$ & $31 / 37$ & $22 / 26$ \\
\hline Date of sampling & $26 / 32$ & $31 / 37$ & $23 / 26$ \\
\hline \multicolumn{4}{|l|}{ Methods } \\
\hline Methods used & $26 / 32$ & $36 / 37$ & $25 / 26$ \\
\hline $\begin{array}{l}\text { Statement about limitations of } \\
\text { repeat sizing }\end{array}$ & $12 / 32$ & $23 / 37$ & $15 / 26$ \\
\hline $\begin{array}{l}\text { Error range of repeat size } \\
\text { determination }\end{array}$ & $26 / 32$ & $11 / 37$ & $20 / 26$ \\
\hline Mean & $\begin{array}{c}25.3 / 32 \\
79 \%\end{array}$ & $\begin{array}{c}31.0 / 37 \\
84 \%\end{array}$ & $\begin{array}{r}19 / 26 \\
73 \%\end{array}$ \\
\hline
\end{tabular}

${ }^{\text {a }}$ These reporting items are not required or recommended by ISO 15189 or the guidelines.

a correct genotype and transcription errors were its reasons. They were more frequent with newly registered laboratories. Other EQA schemes, run by EMQN or other organizations, also consistently identify errors. ${ }^{3,5-8,31-33}$ Annual mean genotyping scores for the SCA scheme were within the range of those found in other EMQN schemes. Irrespective of the cause, misdiagnoses are unacceptable: the implications for patients and their family are severe and, most often, irreversible, as genetic testing is typically performed once in a lifetime.

Not all laboratories determined or reported repeat sizes. Indeed, this is not the reporting policy in all countries. Repeat size, however, is an integral part of genotyping in SCA. It should always be reported, together with its margin of error: it is a measure of technical quality and, in some cases, may signal expansions with reduced penetrance, intervals of uncertain significance or alleles with increased meiotic instability (with its implications for offspring).

Inaccurate sizing was most probably due to the use of inappropriate techniques or formulae and lack of appropriate controls. Sequencing of reference samples, both within the normal and pathological ranges, or the use of certified reference materials should address the latter adequately. The use of techniques such as agarose gel electrophoresis, on its own, is not adequate. Also, sequencing of all samples, as done by two laboratories, is neither needed nor a realistic approach in diagnostic routine.

Another problem became apparent from the imprecise sizing of normal SCA2 and SCA7 alleles. Both loci are very weakly polymorphic, as 22 and 10 CAG repeats, respectively, account for the majority of their alleles. The fact that some laboratories do not report correctly such common alleles suggests a poor knowledge of the scientific literature. The same happened when interpreting an intermediate allele as a pathogenic expansion.

Counting CAG repeats in ATXN3 was an issue in $2004:^{30,17}$ not including the initial sequence, (CAG) $)_{2}$ CAAAAGCAGCAA, led to a systematic underestimation of six repeats. Redefining this issue in the final report has addressed the matter quite adequately in subsequent years.

All laboratories with gross genotyping errors were contacted by EMQN. Quality assessment is an educational exercise, not a punitive action; its aim is to assist laboratories in their continuous effort towards a higher quality of services. Therefore, it was rather discouraging that a third (5/15) of those with inaccurate sizing results did not participate the following years; and for those who did, more than half $(6 / 10)$ provided some results again outside the limits set. Repeated poor performance should be addressed as an important issue, by organizations providing EQA. Annual participation is essential for any laboratories' quality system and improved performance and is also critical for accreditation under ISO 15189. Although it is not straightforward to compare performance among schemes of the same or different providers, and little data are readily accessible for most of the EQA schemes, the results of three different schemes on cystic fibrosis illustrate that genotyping errors declined in successive EQA rounds. ${ }^{4,31}$

Individual, fully interpreted, concise and informative reports should be the standard practice. ${ }^{3,5}$ Fine examples of clear and concise reports were encountered, and its average quality improved over the 3 years; however, a considerable variation was noticed, even if considering that some laboratories were not reporting in their native language. A majority did not seem to be aware or did not report on the limits of the methodology used, as when they failed to discuss the possibility of having missed a large expansion if homoallelic results were found, or not suggesting further testing, for example, TP-PCR (RP-PCR) ${ }^{34}$ or Southern blot. Reporting of a genotype is rather straightforward, as 
compared to interpreting the sizing results. ${ }^{8}$ It is important to state the conclusion unambiguously, with the appropriate recommendations and the implications for family members. Some practices found, as ticking a box (eg 'positive', 'negative'), are not adequate and rather prone to errors. Conclusions must be accessible for non-geneticists, as reports tend to be copied and passed on to different specialists. Essential information, such as at least two identifiers (full name plus date of birth or a unique lab number) and date of report, was not always present or correct. Although there are no general guidelines on style and format, a clear layout and a concise report is important. Some information (as patient and sample identification, results, interpretation) is critical for the efficacy of a report and should not hide in a long letter with heavy supplementary information, as it did in more than a few.

We cannot know if these results reflect the true practices in SCA testing. Although strongly recommended, EQA is voluntary and might be biased towards better performing laboratories. Also, EQA samples are not always treated the same way as routine referrals; many laboratories do not attribute them unique numbers, indicating that they might not have been enrolled in the daily sample pool. Thus, the error rates found may still be overestimating true laboratory performance. The need for EQA was also underlined by the finding that, among 14 laboratories that fully participated over the 3 years, only 2 (14.2\%) achieved maximum genotype scores for all cases.

In conclusion, this EQA has demonstrated to be an important tool to alert laboratories to problems and shortcomings and, in time, will improve laboratory services, in general. Regular participation in EQA will help labs achieving and maintaining proficient testing.

In addition, this scheme emphasized (1) the need for the development of certified reference material and (2) a best practice meeting to discuss the most appropriate methodologies, standardize and improve accurate allele sizing, update normal and pathological reference ranges, and update guidelines for genotyping, analytical and clinical interpretation, and reporting for genetic testing in SCA.

\section{Acknowledgements}

We acknowledge the proficient work of Joana Cerqueira, Paula Magalhães, Isabel Silveira, Carla Martins, Luís Correia, Mia Vercammen and Deborah Vandermaelen.

\section{Notes:}

Electronic-Database Information:

http://www.emqn.org for EMQN (European Molecular Genetics Quality Network)

http://www.eurogentest.org for EuroGentest

http://www.iso.org for ISO 15189: 2007: Medical laboratories particular requirements for quality and competence http://www.ssmg.ch for SSMG (Swiss Society of Medical Genetics), guidelines for reporting 2003.

\section{References}

1 Sequeiros J, Martins S, Silveira I: Epidemiology and population genetics of inherited ataxias; in Subramony SH, Dürr A (eds): Handbook of Clinical Neurology, Ataxic Disorders. Amsterdam: Elsevier (in press).

2 Libeer JC: Role of external quality assurance schemes in assessing and improving quality in medical laboratories. Clin Chim Acta 2001; 309: 173-177.

3 Losekoot M, Bakker B, Laccone F, Stenhouse S, Elles R: A European pilot quality assessment scheme for molecular diagnosis of Huntington's disease. Eur J Med Genet 1999; 7: 217-222.

4 Dequeker E, Ramsden S, Gordy W, Stenzel T, Barton D: Quality control in molecular genetic testing. Nat Rev Genet 2001; 2: 717-723.

5 Mueller CR, Kristoffersson U, Stoppa-Lyonnet D: External quality assessment for mutation detection in the BRCA1 and BRCA2 genes: EMQN's experience of 3 years. Ann Oncol 2004; 15 (Suppl 1): i14-i17.

6 Hertzberg M, Neville S, Favaloro E, McDonald D: External quality assurance of DNA testing for thrombophilia mutations. Am J Clin Pathol 2005; 123: 189-193.

7 Hertzberg M, Neville S, McDonald D: External quality assurance of molecular analysis of haemochromatosis gene mutations. J Clin Pathol 2006; 59: 744-747.

8 Ramsden S, Deans Z, Robinson D et al: Monitoring standards for molecular genetic testing in the United Kingdom, The Netherlands, and Ireland. Genet Test 2006; 10: 147-156.

9 Orr HT, Chung MY, Banfi S, Schwinger E, Bark K: Expansion of an unstable trinucleotide CAG repeat in spinocerebellar ataxia type 1. Nat Genet 1993; 4: 221-226.

10 Zühlke C, Dalski A, Hellenbroich Y, Bubel S, Schwinger E, Burk K: Spinocerebellar ataxia type 1 (SCA1): phenotype-genotype correlation studies in intermediate alleles. Eur J Hum Genet 2002; 10: 204-209.

11 Koide R, Ikeuchi T, Onodera $\mathrm{O}$ et al: Unstable expansion of CAG repeat in hereditary dentatorubral-pallidoluysian atrophy (DRPLA). Nat Genet 1994; 6: 9-13.

12 Nagafuchi S, Yanagisawa $\mathrm{H}$, Sato $\mathrm{K}$ et al: Dentatorubral and pallidoluysian atrophy expansion of an unstable CAG trinucleotide on chromosome 12p. Nat Genet 1994; 6: 14-18.

13 Imbert G, Saudou F, Yvert G et al: Cloning of the gene for spinocerebellar ataxia 2 reveals a locus with high sensitivity to expanded CAG/glutamine repeats. Nat Genet 1996; 14: 285-291.

14 Pulst S-M, Nechiporuk A, Nechiporuk T et al: Moderate expansion of normally biallelic trinucleotide repeat in spinocerebellar ataxia type 2. Nat Genet 1996; 14: 269-275.

15 Sanpei K, Takano H, Igarashi $\mathrm{S}$ et al: Identification of the spinocerebellar ataxia type 2 gene using a direct identification of repeat expansion and cloning technique, DIRECT. Nat Genet 1996; 14: 277-283.

16 Silveira I, Miranda C, Guimarães L et al: Trinucleotide repeats in 202 families with ataxia: a small expanded (CAG)n allele at the SCA17 locus. Arch Neurol 2002; 59: 623-629.

17 Maciel P, Gaspar C, DeStefano AL et al: Correlation between CAG repeat length and clinical features in Machado-Joseph disease. Am J Hum Genet 1995; 57: 54-61.

18 Maciel P, Costa MC, Ferro A et al: Improvement in the molecular diagnosis of Machado-Joseph disease. Arch Neurol 2001; 58: 1821-1827.

19 Takiyama Y, Sakoe K, Nakano I, Nishizawa M: Machado-Joseph disease: cerebellar ataxia and autonomic dysfunction in a patient with the shortest known expanded allele (56 CAG repeat units) of the MJD1 gene. Neurology 1997; 49: 604-606.

20 Padiath QS, Srivastava AK, Roy S, Jain S, Brahmachari SK: Identification of a novel 45 repeat unstable allele associated with a disease phenotype at the MJD1/SCA3 locus. Am J Med Genet B 2005; 133: 124-126. 
21 Zhuchenko O, Bailey J, Bonnen $\mathrm{P}$ et al: Autosomal dominant cerebellar ataxia (SCA6) associated with small polyglutamine expansions in the alpha 1A-voltage-dependent calcium channel. Nat Genet 1997; 15: 62-69.

22 David G, Abbas N, Stevanin G et al: Cloning of the SCA7 gene reveals a highly unstable CAG repeat expansion. Nat Genet 1997; 17: $65-70$.

23 Stevanin G, Giunti P, David G et al: De novo expansion of intermediate alleles in spinocerebellar ataxia type 7. Hum $\mathrm{Mol}$ Genet 1998; 11: 1809-1813.

24 Koob MD, Moseley ML, Schut LJ et al: An untranslated CTG expansion causes a novel form of spinocerebellar ataxia (SCA8). Nat Genet 1999; 21: 379-384.

25 Matsuura T, Yamagata T, Burgess DL et al: Large expansion of the ATTCT pentanucleotide repeat in spinocerebellar ataxia type 10 . Nat Genet 2000; 26: 191-194.

26 Alonso I, Jardim LB, Artigalas O et al: Reduced penetrance of intermediate size alleles in spinocerebellar ataxia type 10 . Neurology 2006; 66: 1602-1604.

27 Holmes SE, O'Hearn EE, McInnis MG et al: Expansion of a novel CAG trinucleotide repeat in the 5'region of PPP2R2B is associated with SCA12. Nat Genet 1999; 23: 391-392.

28 Zühlke C, Gehlken U, Hellenbroich Y, Schwinger E, Burk E: Phenotypical variability of expanded alleles in the TATA-binding protein gene. Reduced penetrance in SCA17? I Neurol 2003; 250 $161-163$.

29 Oda M, Maruyama H, Komure O et al: Possible reduced penetrance of expansion of 44 to $47 \mathrm{CAG} / \mathrm{CAA}$ repeats in the TATA-binding protein gene in spinocerebellar ataxia type 17. Arch Neurol 2004; 61: 209-212.

30 Kawaguchi Y, Okamoto T, Taniwaki M et al: CAG expansions in a novel gene for Machado-Joseph disease at chromosome 14q32.1. Nat Genet 1994; 8: 221-222.

31 Dequeker E, Cassiman JJ: Evaluation of CFTR gene mutation testing methods in 136 diagnostic laboratories: report of a large European external quality assessment. Eur J Hum Genet 1998; 6: $165-175$.

32 Noll WW, Belloni DR, Stenzel T, Gordy WW: HFE C282Y heterozygote with intron 4 (5569A) polymorphism was correctly genotyped by all 67 laboratories in a nationwide ACMG/CAP molecular genetics proficiency survey. Nat Genet 1999; 23: $271-272$.

33 Lutz CT, Foster PA, Noll WW et al: Multicenter evaluation of PCR methods for the detection of factor V Leiden (R506Q) genotypes. Clin Chem 1998; 44: 1356-1358.

34 Warner JP, Barron LH, Goudie D et al: A general method for the detection of large CAG repeat expansions by fluorescent PCR. J Med Genet 1996; 33: 1022-1026. 